\title{
MINIREVIEW
}

\section{Regeneration review reprise}

\author{
Jessica L Whited and Clifford J Tabin* \\ See research articles http://www.biomedcentral.com/1741-7007/7/83 and http://www.biomedcentral.com/1741-7007/8/5
}

\begin{abstract}
There have been notable advances in the scientific understanding of regeneration within the past year alone, including two recently published in BMC Biology. Increasingly, progress in the regeneration field is being inspired by comparisons with stem cell biology and enabled by newly developed techniques that allow simultaneous examination of thousands of genes and proteins.
\end{abstract}

Regeneration of complex structures such as appendages has long fascinated and perplexed biologists. Historically, understanding regeneration has been approached, often to great effect, through surgical operations, grafting, dissections, and other tools borrowed from embryologists. More recently, additional insights have been gleaned through analyzing appendage development genes during regeneration. Now, rather than simply investigating the potential roles of genes already known to be important in the context of the embryonic development of a structure, researchers are using modern techniques to interrogate gene transcription and protein translation during various stages of regeneration itself [1-4]. In addition, new clues are being drawn from other areas of biology to tackle longstanding regeneration questions $[1,2,5]$.

A year ago we [6] provided a general outline of the morphological events accompanying limb regeneration and a summary of the classical experiments that provided some of the most salient principles of regeneration; these aspects are therefore not extensively reviewed here. Instead, the focus is on what has been learned within the past year and what discoveries might be awaiting fresh perspectives and tools on the horizon.

*Correspondence: tabin@genetics.med.harvard.edu

Department of Genetics, Harvard Medical School, 77 Avenue Louis Pasteur, NRB 360, Boston, MA 02115, USA.
The cellular origin of the blastema and the lineage of regenerating cells

From the early days of regeneration study, a major goal has been to characterize the cellular and molecular nature of blastema cells. The blastema is the structure that develops at the cut end of an amputated limb in some vertebrates, from which the limb regenerates. Great debate has ensued over where these cells come from, that is, from which tissues they arise. In salamanders, a favorite method has been to create chimeric animals in which a population of cells from a donor, distinguishable in some way, is introduced into a host. The donor cells are followed over the course of regeneration. 2009 saw the publication of a lineage study of the regenerating salamander limb using the most sophisticated labeling method yet, transgenesis [7]. Cells from transgenic animals expressing green fluorescent protein (GFP) were transplanted into non-GFP-expressing host embryos. The chimeras were allowed to develop, their limbs were amputated, and the journeys of GFP-expressing cells through the blastema and out into the new limb were documented. The analysis revealed very little transdifferentiation between cell types, although not all cell types could be analyzed in this manner for technical reasons. This study [7] suggested that the blastema is not a homogeneous population of molecularly identical cells but is instead heterogeneous from its inception, a conclusion that challenges the notion that complete dedifferentiation is a major force behind blastema creation (Figure 1). Instead, a new model has emerged in which some amount of dedifferentiation occurs - but perhaps only enough to send cells 'backwards in time' yet not enough to make them completely naïve. At minimum, there seem to be two distinct cell populations in the blastema giving rise, respectively, to muscle and nonmuscle tissue (cartilage, fibroblasts, and connective tissue), a situation at least analogous to that found in the early developing limb bud [7].

\section{New ways of comparing regeneration with other biological processes}

Parallels can be drawn between blastema cells and stem cells. Although blastema cells are like stem cells insofar as 


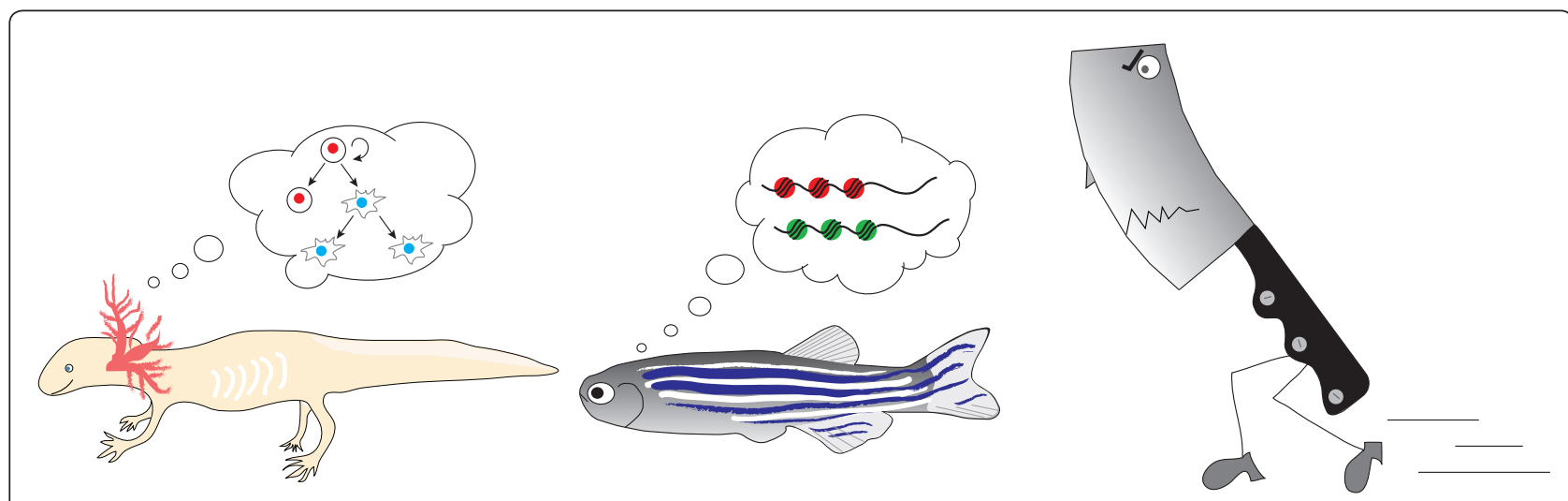

Figure 1. The limbs of amphibians (left) and the fins of zebrafish (middle) both regenerate when amputated (right). Recent work in salamanders [1] has uncovered some similarities between cells undergoing regeneration and stem cells. In zebrafish [5], histone methylation/ demethylation may have a key role in controlling gene expression during fin regeneration.

they alone can replace an entire structure, the recent lineage data might suggest that blastema cells share attributes with stem cells but that any individual blastema cell is not pluripotent for replacing the lost limb. Recently, a protocol for creating pluripotent stem cells (induced pluripotent stem cells, iPSCs) from differentiated cells, through the expression of just a handful of genes, has been developed [8]. A blastema cell might be predicted to be molecularly similar to a cell somewhere along this trajectory of being driven into a more undifferentiated state even if it never really becomes completely pluripotent (and thus would more properly be referred to as a multipotent progenitor cell than a stem cell).

Two studies have considered this hypothesis. Maki et al. [1] found the expression of three of the core iPSC genes Sox 2, Klf4, and $c-m y c$ - to be upregulated in regenerating newt lens and limb. Christen et al. [2] explored the concept of blastema cells behaving as cells on the iPSC trajectory. In regenerating zebrafish fins, homologs of genes associated with acquisition of pluripotency were found to be expressed. Further experimentation revealed that a few of these genes are even necessary for regeneration to occur. However, not all of the 'stem cell genes' analyzed were expressed at appreciable levels in regenerating tissues, and many showed much higher levels of expression in the tissues that are thought to approximate embryonic stem cells from the relevant species. Among blastema cells, 'pluripotent embryonic reference cells' (from the same species), and iPSCs (created in vitro), the pluripotent embryonic reference cells and the iPSCs probably have more in common from a gene expression standpoint than blastema cells have to either of them. Perhaps this is not unexpected because we know the pluripotent embryonic reference cells and the iPSCs have more developmental potential than the blastema cells, that is, a blastema cell does not naturally replace an entire organism, while a whole mouse can be cloned from an iPSC.

Another issue is that when dissecting a tissue to use as the zero-hour time point in the zebrafish experiments, there is the possibility of recovering some actual stem cells among the tissue harvested. There exists the possibility that regeneration of appendages is supported at least in part by a local population of stem cells. If some stem cells are among the cells in the sample, they may be contributing to the high levels of expression of some 'stem cell genes' in the intact tissue.

Approximating loss-of-function of two of the 'stem cell genes' (pou and sox2) using morpholino oligonucleotides caused impaired regeneration in tail fins; and yet, these genes are among those expressed in intact tail fins at levels on a par with or higher than those found in blastemas. The authors [2] conclude that blastema cells in zebrafish fins and Xenopus limbs are not completely analogous to induced pluripotent mammalian stem cells but they share some similarities in gene expression. Perhaps organisms capable of epimorphic regeneration maintain expression of pluripotency factors in otherwise differentiated tissue as a way to ready themselves to regenerate should the need arise.

Another similarity between embryonic stem cells and cells undergoing regeneration relates to the epigenetic status of their chromatin. A recent study of zebrafish tail fins [5] (Figure 1) identified targets of histone methylases, and among these targets were the promoters of many key patterning genes expressed during regeneration. Like embryonic stem cells, cells capable of undergoing epimorphic regeneration might use these histone modifications to keep cells in a poised state whereby they can easily turn 'on' or 'off' the expression of key genes. For example, repressive chromatin modifications seem to be removed from the promoters of genes required during fin 
regeneration, allowing their expression. Given that many of the required genes are targets of the same methylases and demethylases, modulating the expression or activity of the methylases or demethylases themselves could be an efficient means of controlling a whole suite of regeneration genes. Indeed, loss of one of the demethylases (Kdm6b.1) results in inhibition of tail regeneration, perhaps because the promoter for one of its targets $(d l x 4 a$, a homeobox gene whose family members are involved in appendage development across many taxa) cannot be activated [5].

\section{Looking directly at regeneration}

Another way to gain insight into regenerative processes is to simply ask which genes are active and which proteins are made, rather than selecting candidates for study on the basis of roles in potentially related processes. This type of unbiased approach to characterizing protein composition in regenerating Xenopus tadpole limb, performed by King, Neff, and Mescher [3], uncovered upregulation of immune system proteins and stem cell proteins. Another proteomics study, performed by Rao and colleagues [4], revealed potential roles for previously unimplicated protein networks in axolotl limb regeneration. For example, a change in cytosolic $\mathrm{Ca}^{2+}$ levels might be one of the earliest cellular events following amputation, as evidenced by the upregulation of an enzyme required for the synthesis of a precursor to the inositol triphosphate/diacylglycerol signaling pathway, which modulates cytosolic $\mathrm{Ca}^{2+}$ stores. Another signaling pathway that may have an important role immediately after amputation is the nitric oxide (NO) signaling pathway - the enzyme that synthesizes NO, NOS1, was upregulated more than any other identified protein at 1 day after amputation. Although almost nothing is known about a potential role for NO signaling in limb regeneration, the fact that the nitric oxide synthase (NOS1) peptide was found in this study [4] is satisfying, because a previous study had found the gene encoding it to be upregulated in Xenopus limb buds at a stage supportive of regeneration but not at a stage when regeneration could not occur [9]. These two, and other, pathways implicated in this proteomic study [4] can serve as pointers for future functional studies.

\section{A new perspective on amphibian and mammalian regeneration}

The de novo approach to understanding limb regeneration led to the identification of a proximallydistally graded cell-surface protein, Prod1, and eventually its ligand, the newt Anterior gradient protein, in the past decade. Last year the Prod1 crystal structure was solved [10] and a surprising conclusion was reached: the protein is not homologous to any known mammalian proteins, and, along with its close relatives, is suspected to be a salamander-specific innovation. The conclusion is notable because it might support a paradigm shift in the regeneration field. Previously, many researchers favored a theory that epimorphic regeneration of body parts was an ancestral trait common to all animals that had been extinguished in some lineages. Thus, re-activating a regenerative process that might lie latent seemed a reasonable way to improve regenerative prospects in humans once the key molecular events were elucidated in animals that retain regenerative capabilities. If, however, Prod1 is indeed key, and necessary, in limb regeneration, but mammals do not possess it, the outlook must be revised. This realization might lead to more effort being brought to bear on the question of regeneration itself, rather than in comparison with development, or to stem cells, or to any other worthy but potentially limiting paradigm. The coming decade promises to be an exciting time for unraveling the secrets to regeneration.

\section{Acknowledgements}

Work on regeneration in the Tabin lab is supported by grants from the $\mathrm{NIH}$.

Published: 16 February 2010

\section{References}

1. Maki N, Suetsugu-Maki R, Tarui H, Agata K, Del Rio-Tsonis K, Tsonis PA: Expression of stem cell pluripotency factors during regeneration in newts. Dev Dyn 2009, 238:1613-1616.

2. Christen B, Robles V, Raya M, Paramonov I, Izpisua Belmonte JC: Regeneration and reprogramming compared. BMC Bio/ 2010, 8:5.

3. King MW, Neff AW, Mescher AL: Proteomics analysis of regenerating amphibian limbs: changes during the onset of regeneration. Int J Dev Biol 2009, 53:955-969.

4. Rao N, Jhamb D, Milner DJ, Li B, Song F, Wang M, Voss SR, Palakal M, King MW, Saranjami B, Nye HL, Cameron JA, Stocum DL: Proteomic analysis of blastema formation in regenerating axolotl limbs. BMC Bio/ 2009, 7:83.

5. Stewart S, Tsun ZY, Izpisua Belmonte JC: A histone demethylase is necessary for regeneration in zebrafish. Proc Natl Acad Sci USA 2009, 106:19889-19894.

6. Whited JL, Tabin CJ: Limb regeneration revisited. J Bio/ 2009 8:5.

7. Kragl M, Knapp D, Nacu E, Khattak S, Maden M, Epperlein HH, Tanaka EM: Cells keep a memory of their tissue origin during axolotl limb regeneration. Nature 2009, 460:60-65.

8. Takahashi K, Yamanaka, S: Induction of pluripotent stem cells from mouse embryonic and adult fibroblast cultures by defined factors. Cell 2006, 126:663-676.

9. Grow M, Neff AW, Mescher AL, King MW: Global analysis of gene expression in Xenopus hindlimbs during stage-dependent complete and incomplete regeneration. Dev Dyn 2006, 235:2667-2685.

10. Garza-Garcias A, Harris R, Esposito D, Gates PB, Driscoll PC: Solution structure and phylogenetics of Prod1, a member of the three-finger protein superfamily implicated in salamander limb regeneration. PLoS One 2009, 4:e7123.

See other regeneration and stem cell articles http://jbiol.com/content/8/8/70 and http://jbiol.com/content/9/2/14

doi:10.1186/jbiol224

Cite this article as: Whited $J \mathrm{~L}$ and Tabin CJ: Regeneration review reprise. Journal of Biology 2010, 9:15. 